\title{
Blockage of bone marrow kinase in chromosome $X$ enhances ABC294640-induced growth inhibition and apoptosis of colorectal cancer cells
}

\author{
Ruifang Jin, Zhoufeng Chen*, Haibo Xue, Mengjun Chen \\ Department of Gastroenterology, The First Affiliated Hospital of Wenzhou Medical University, Wenzhou, 325000, China
}

${ }^{*}$ For correspondence: Email: chenzhoufcwz@163.com

Sent for review: 5 January 2018

Revised accepted: 25 April 2018

\begin{abstract}
Purpose: To investigate the role of bone marrow kinase in chromosome $X(B M X)$ in colorectal cancer (CRC) cell resistance to $A B C 294640$ treatment.

Methods: HCT-116R, LS174T and WiDr cells were transfected with either BMX-specific siRNA or scrambled siRNA, and then BMX mRNA and protein expressions were detected by quantitative polymerase chain reaction ( $q P C R$ ) and western blotting, respectively. The cells were treated with ABC294640 and cell viability evaluated using cell counting and colony formation assays. Apoptosis was determined by detecting caspase 3/7 activity. To evaluate tumor growth of HCT-116R cells, a xenograft model was utilized to measure tumor size.

Results: Pharmacological inhibition of sphingosine kinase type 2 (SK2) with ABC294640 significantly decreased cell viability $(p<0.001)$ when compared with control group. SK2 inhibition also remarkably induced apoptosis in HCT-116 CRC cells in a dose-dependent manner $(p<0.01$ and $p<0.001)$. However, no significant effects were observed in HCT-116R, LS174T, or WiDr cells following ABC294640 treatment. BMX mRNA and protein expression increased in ABC294640-resistant cell lines. In addition, silencing BMX expression with siRNA potentiated ABC294640-induced inhibition of tumor growth in $C R C$ cells in vitro and in vivo.

Conclusion: ABC294640-induced BMX upregulation impedes the antitumor effect of $A B C 294640$ in CRC cells. Therefore, these results may provide a novel therapeutic strategy for CRC using a combination of ABC294640 treatment and BMX blockade.
\end{abstract}

Keywords: ABC294640, Apoptosis, Bone marrow kinase in chromosome X, Cell viability, Colorectal cancer

\begin{abstract}
This is an Open Access article that uses a funding model which does not charge readers or their institutions for access and distributed under the terms of the Creative Commons Attribution License (http://creativecommons.org/licenses/by/4.0) and the Budapest Open Access Initiative (http://www.budapestopenaccessinitiative.org/read), which permit unrestricted use, distribution, and reproduction in any medium, provided the original work is properly credited.

Tropical Journal of Pharmaceutical Research is indexed by Science Citation Index (SciSearch), Scopus, International Pharmaceutical Abstract, Chemical Abstracts, Embase, Index Copernicus, EBSCO, African Index Medicus, JournalSeek, Journal Citation Reports/Science Edition, Directory of Open Access Journals (DOAJ), African Journal Online, Bioline International, Open-J-Gate and Pharmacy Abstracts
\end{abstract}

\section{INTRODUCTION}

Colorectal cancer (CRC) is one of the most common malignancies, ranking second in incidence and mortality among all types of malignant worldwide [1]. Without reliable biomarkers, diagnosis of CRC usually occurs in the advanced stage, leading to high mortality in these patients. Currently, surgery and conventional chemotherapy remain the primary treatments for CRC [2]. However, tumor relapse and/or metastasis severely limit their clinical 
effectiveness, which demonstrates that finding effective cancer therapies remains an important medical challenge [3]. Therefore, more investigation of mechanisms of CRC pathogenesis is needed to develop effective treatments for CRC.

Sphingosine 1-phosphate (S1P) is a pleiotropic molecule and involved in various intracellular functions, including cell survival, cell motility, and angiogenesis [4]. S1P is formed via sphingosine phosphorylation by sphingosine kinase type 1 (SK1) and sphingosine kinase type 2 (SK2) [5]. The two kinases are highly conserved but have different subcellular localizations functions, and pharmacology [6]. Initially, overexpression of SK2 was reported to induce apoptosis, mediated by its $\mathrm{BH} 3$ domain [7]. Subsequently, it was found that SK2 downregulation had dramatic antitumor function in several tumor types, including colon cancer [8]. Loss of SK2 had stronger anticancer effects than suppression of SK1, providing a rationale for targeting SK2 in cancer therapy [9]. ABC294640 is an inhibitor of SK2 which showed very good bioavailability when administered orally as well as a favorable safety profile in various pre-clinical models [10, 11]. However, the drug resistance of cancer cell to ABC294640 is not clear.

Bone marrow kinase in chromosome $X(B M X)$ is a member of Tec family [12]. It has two critical domains, a $\mathrm{PH}$-like domain that mediates membrane binding and an $\mathrm{SH} 2$ domain important for the binding of tyrosine-phosphorylated proteins [13]. BMX is regulated by several oncogenes such as Src and phosphoinositide 3kinase (PI3K), and is activated by inflammatory pathways [14]. It been implicated in tumor progression in prostate cancer, bladder cancer, and glioblastoma [15]. Additionally, BMX is reported to be involved in drug resistance. For example, overexpression of BMX abolished the miR-495-induced inhibition of drug resistance [16]. In addition, it suppressed the ephrin receptor A3 (EPHA3)-induced drug sensitivity of small cell lung cancer [17]. In the present study, the effect of $B M X$ on the $C R C$ response to ABC294640 treatment was explored.

\section{EXPERIMENTAL}

\section{siRNA and transfection}

HCT-116R, LS174T and WiDr cells $\left(3 \times 10^{5}\right.$ cells/well) were cultured in 6-well plates overnight. Then the cells were transfected with either BMX-specific siRNA or scrambled siRNA (30 nM; Ribo-Bio, China) using lipofectamine 3000 (Thermo Fisher Scientific, Waltham, MA,
USA) according to the manufacturer's instructions. The protein and RNA were extracted after transfection. BMX siRNA sequences were as follows: GUACCAGUCUAGCGCAAUAUU (sense) and UAUUGCGCUAGACUGGUACUU (anti-sense).

\section{Quantitative RT-PCR (qPCR)}

In accordance with the specifications, total RNA was isolated using TRIzol reagent (Invitrogen). Reverse transcription of RNA was carried out with a first strand cDNA kit (Sigma, Munich, Germany) followed by PCR amplification with a SYBR Green PCR kit (Thermo Fisher Scientific) run on an $\mathrm{ABI} 7300$ Thermocycler (Thermo Fisher Scientific). The primers were as following: $\beta$-actin Forward: 5'-AAGGCCAACCGCGAGAAG AT-3'; and Reverse:5'-TGATGACCTGGCCGTC AGG-3'; BMX Forward:5'-GAGCCGAAGTCA GTGGTTGA-3'; and Reverse: 5'-ACTTCCCGTC CACGAAGAAC-3'. Differences in relative mRNA expression were calculated with the $2^{-\Delta \Delta C t}$ method [18].

\section{Caspase $3 / 7$ activity assay}

For detection of apoptosis, caspase-Glo® $3 / 7$ Assay kit from Promega was used. Cells $\left(5 \times 10^{3}\right.$ cells/well) were cultured in 96-well plates and treated with either ABC294640 (0.3 or $3 \mu \mathrm{M})$ or left untreated for $48 \mathrm{hr}$. The Caspase-Glo® 3/7 Reagent was added directly into the wells followed by the luminometer readings.

\section{Protein isolation and western blot analysis}

Cells were lysed with ice cell lysis buffer from cell Signaling Technology (Danvers, MA, USA). Protein was quantified using a bicinchoninic acid assay kit (EMD Millipore, Billerica, MA, USA). Total protein was subjected to $10 \%$ SDS polyacrylamide gel electrophoresis and were transferred onto PVDF membranes. Membranes were probed with specific antibodies at $4{ }^{\circ} \mathrm{C}$ overnight (anti-GAPDH, 1:5000 dilution, \#D16H11; anti-BMX, 1:1000 dilution, \#24773, Cell Signaling Technology). The membranes were then incubated with secondary antibodies at room temperature for $2 \mathrm{~h}$ (1:5,000 dilution; \#7074, Cell Signaling Technology). Bands were visualized using RapidStep ${ }^{\mathrm{TM}}$ ECL Reagent (EMD Millipore).

\section{Tumor xenografts}

Six-week old nude mice (Charles River Laboratories) received a single subcutaneous injection of $1 \times 10^{6}$ HCT-116R cells in the right flank. Tumor volumes were assessed with digital 
calipers and calculated as previously described [19]. Tumors were measured 3 times weekly, including total mouse weights. At the end of the study, the tumors were excised, and weighed. The study was approved by Animal Ethic Committee of the First Affiliated Hospital of Wenzhou Medical University (approval ref no. 2017-0003), and were in accordance with "Principles of Laboratory Animal Care" (NIH publication no. 85 - 23, revised 1985) [19].

\section{RESULTS}

\section{Effect of ABC294640 on CRC cells}

Cytotoxicity assays demonstrated that ABC294640 significantly decreased cell viability in a dose-dependent fashion in HCT-116 cells. In contrast, ABC294640 had marginal effect on cell viability in LS174T and WiDr cells, with less than 10 -fold change in viability $(p<0.001$; Figure $1 \mathrm{~A})$. In addition to cytotoxicity, apoptosis was evaluated in the three CRC cell lines following ABC294640 treatment. ABC294640 treated HCT-116 cells displayed higher caspase $3 / 7$ activity relative to the untreated group $(p<0.001$ and $p<0.0001$; Figure 1B). However, ABC294640 treatment had no effect on apoptosis in LS174T and WiDr cells (Figure 1B). These results demonstrate that HCT-116 cells are sensitive to ABC294640 treatment whereas LS174T and WiDr cells are more resistant.

\section{Upregulation of BMX in CRC cells associated with ABC294640 sensitivity}

To reveal the mechanism underlying drug resistance, the ABC294640-resistant cell line, HCT-116R, was established from HCT-116 cells by continuous passage in the presence of sublethal doses of ABC294640. The drug

A

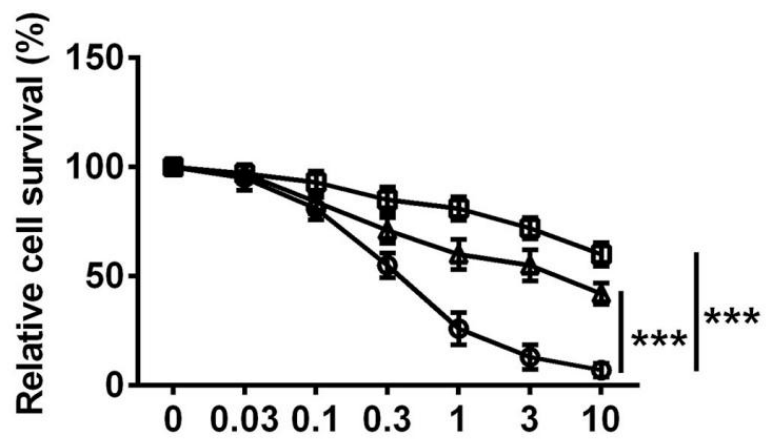

sensitivities of HCT-116R and the parental HCT116 cells to $A B C 294640$ were determined using the CCK8 cytotoxicity and caspase $3 / 7$ assays. As shown in Figure $2 \mathrm{~A}$, the half maximal inhibitory concentration of $\mathrm{ABC} 294640$ treatment in HCT-116 cells was $260 \mathrm{nM}$, whereas ABC294640 did not affect HCT-116R cell viability $(p<0.001)$. Consistently, ABC294640 increased the level of caspase $3 / 7$ activity in HCT- 116 cells $p<0.001$ and $p<0.0001$ while showing no effect in HCT-116R cells (Figure $2 \mathrm{~B}$ ).

To investigate the effect of $B M X$ on $C R C$ resistance to $A B C 294640$, the expression of BMX was evaluated in drug-resistant cells and drug sensitive cells by GPCR and Western blotting. Both protein and mRNA expression levels of BMX were significantly higher in HCT116R, LS174T, and WiDr compared to HCT-116 cells $(p<0.0001$; Figure $2 \mathrm{C}$ and D).

\section{Inhibition of BMX augment ABC294640 potency in CRC}

To clarify the role of $B M X$ in CRC resistance to ABC294640, HCT-116R, LS174T and WiDr cells were treated with $A B C 294640$ in the presence or absence of siRNA against BMX (siBMX). Cotreatment with siBMX and ABC294640 significantly inhibited the cell survival of $C R C$ compared to each single treatment group ( $p<$ 0.001 and $p<0.0001$; Figure $3 \mathrm{~A})$. In addition $A B C 294640$ treatment $(3 \mu M)$ and siBMX induced apoptosis of CRC cells $48 \mathrm{~h}$ posttreatment. Combinational treatment of ABC294640 and siBMX dramatically enhanced apoptosis in CRC cells $(p<0.001$ and $p<$ 0.0001 ; Figure $3 \mathrm{~B}$ ). The results indicate that $\mathrm{BMX}$ is resistance molecule during ABC294640 treatment, and specific inhibition of $B M X$ enhanced ABC294640 sensitivity in CRC cells.
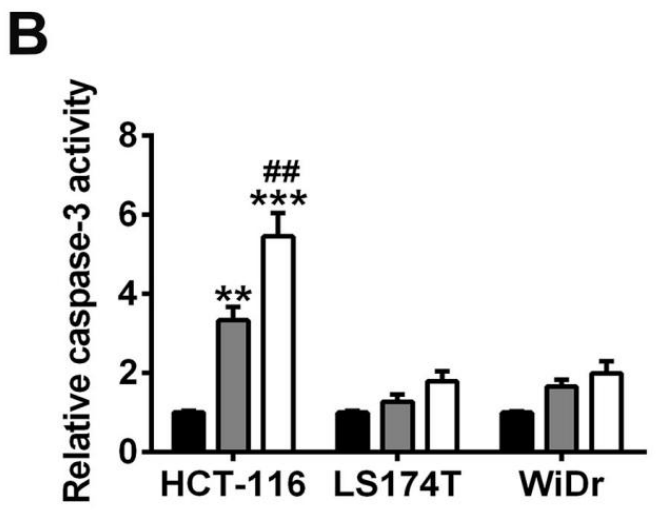

Figure 1: (A) Impact of ABC294640 treatment on CRC cell viability. HCT-116, LS174T, and WiDr cells were treated with $\mathrm{ABC} 294640(0-10 \mu \mathrm{M})$ for $72 \mathrm{~h}$ and cell survival was assessed. Relative cell survival rate was represented as a percentage of control group. (B) Effect of ABC294640 on caspase 3/7 activity in CRC cells. Cells were incubated with ABC294640 at 0.3 and $1 \mu \mathrm{M}$ and caspase $3 / 7$ activity evaluated. DMSO treatment was

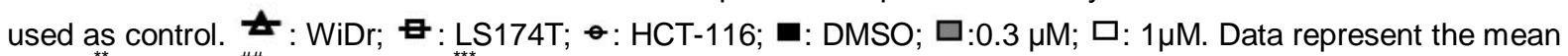
\pm SD. ${ }^{* *} p<0.01,{ }^{\# \#} p<0.01$, and ${ }^{* \star *} p<0.001$ 
A

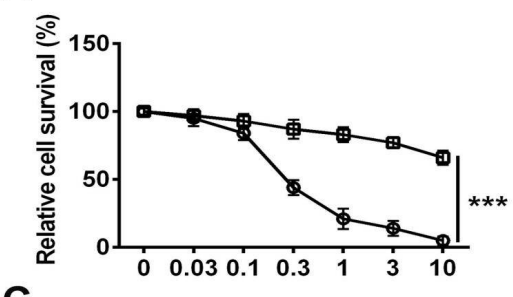

C

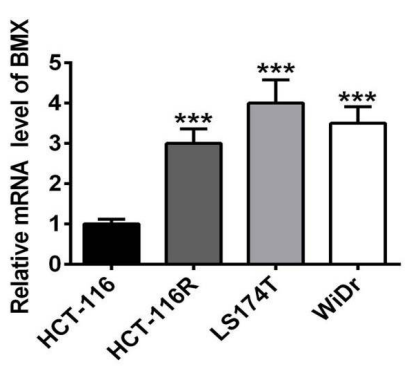

B

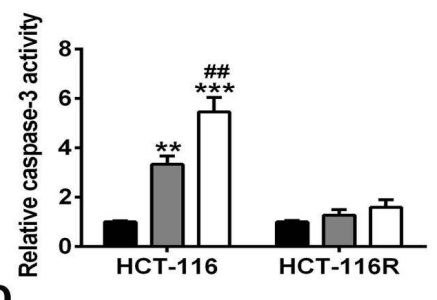

D

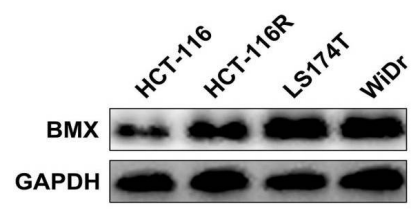

Figure 2: Upregulation of BMX associated with ABC294640 sensitivity in CRC. (A) Effect of ABC294640 on HCT-166 and HCT-166R cell viability. HCT-116 and HCT-166R cells were incubated with ABC294640 $(0-10 \mu \mathrm{M})$ for $72 \mathrm{~h}$ and cell survival was determined. (B) Effect of ABC294640 on caspase 3/7 activity in HCT-166 and HCT166R. Cells were incubated with $A B C 294640$ at 0.3 and $1 \mu \mathrm{M}$ and caspase $3 / 7$ activity was evaluated. (C) mRNA levels of BMX in CRC cells were measured by QPCR. (D) BMX protein expression was measured by western blot in CRC cells. : HCT-166R; : HCT-116; $\square$ : DMSO; $\square: 0.3 \mu \mathrm{M}$; $\square$ : $1 \mu \mathrm{M}$. Data represent mean \pm SD. ${ }^{*} p<$ $0.01, \quad p<0.001$, and ${ }^{\# \#} p<0.01$

A

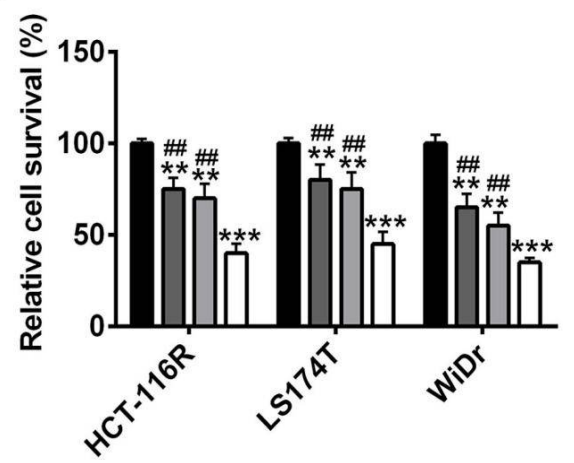

B

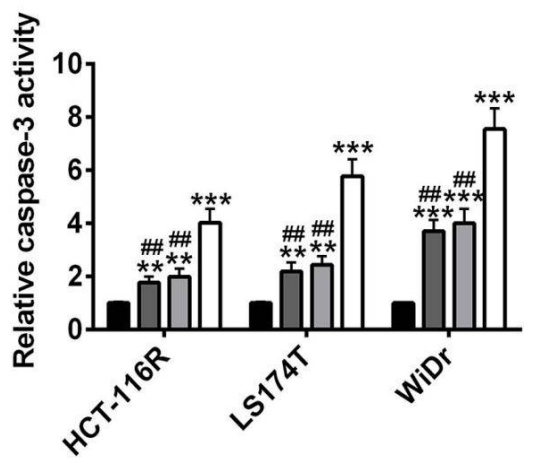

Figure 3: Inhibition of BMX increased $A B C 294640$ potency in vitro. $C R C$ cells were incubated with $A B C 294640$ $(3 \mu \mathrm{M})$ in the absence and presence of BMX siRNA. Cell survival $(A)$ and apoptosis (B) were determined by CCK8 assay and caspase 3/7 activity assay, respectively. $\mathbf{~ : ~ D M S O ; ~} \square$ : ABC294640 $3 \mu \mathrm{M}$; $\square$ : siBMX; $\square$ : $A B C+$ siBMX. Data represent mean \pm SD. ${ }^{* *} p<0.01,{ }^{* * *} p<0.001$, and ${ }^{\# \#} p<0.01$

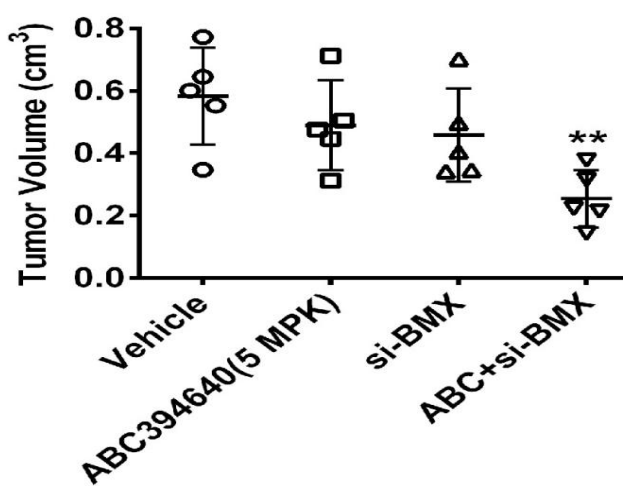

Figure 4: Inhibition of BMX increased ABC294640 potency in vivo. HCT-116R cells or HCT-116R in combination with siBMX was injected into the right flanks of nude mice to induce tumors. Tumors were measured with digital calipers to obtain an average size of $100-150 \mathrm{~mm}^{3}$ followed by randomization into treatment groups. The mice were orally administrated $0.5 \% \mathrm{MC}$ (vehicle control) or ABC294640 at $5 \mathrm{mpk}$, once per day, respectively. Tumors were measured 3 times weekly. Data represent mean \pm SD. ${ }^{* *} p<0.01$

\section{BMX inhibition increase ABC294640 potency in CRC in vivo}

As ABC294640 treatment and knockdown of BMX showed an additive effect in vitro in CRC, it was investigated that whether this also occurred in vivo. As indicated in Figure 4, neither $A B C 294640$ treatment nor siBMX influenced the HCT-116R CRC xenografts in nude mice when given alone. However, administration of $\mathrm{ABC} 294640$ at $5 \mathrm{mpk}$ in combination with siBMX suppressed the growth of HCT-116R tumors $(p<$ $0.01)$. 


\section{DISCUSSION}

There S1P rheostat determines the fate of tumor cells. Thus far, two isoforms of SK, SK1 and SK2, have been identified in humans [19]. It has been shown that cell proliferation and migration was suppressed by downregulation of SK2, which had a greater affect than SK1 [8]. Recently, phase I clinical data for ABC294640 treatment of solid tumors, a highly specific SK2 inhibitor, have demonstrated variable clinical activity, which may reflect either the lack of biomarkers for patient selection and/or deficiency of knowledge about drug-resistant mechanisms [20]. In the current study, the molecular target that contributes to the limited therapeutic efficacy of $A B C 294640$ in $C R C$ was investigated.

ABC294640 has been extensively studied for the treatment of many human tumor types and animal tumor models including CRC. ABC294640 suppressed cell proliferation and induced cell apoptosis in CRC cells in vitro. ABC294640 treatment remarkably inhibited HT29 tumor growth in vivo, suggesting that ABC294640 might be an effective anti-tumor drug in CRC [21]. In the current study, ABC294640 sensitivity in several CRC cell lines was tested, with variable effects among these cell lines, consistent with its clinical activity. This indicates the presence of intrinsic CRC resistance to $\mathrm{ABC} 294640$.

Bone marrow kinase in chromosome $X$ is reported to be overexpressed in various tumor types [22]. It also participates in drug resistance as it suppresses the apoptotic regulator BAK activation via phosphorylation, which facilitates the survival of cells subjected to cytotoxic agents [23]. Bone marrow kinase in chromosome $X$ also is suggested to protect prostate cancer cells from photodynamic induced apoptosis [24]. In addition, suppressing BMX expression reduced the chemo-resistance of H69/AR cells [25]. In this study, BMX mRNA and protein expression were increased in ABC294640-resistant CRC cells and silencing $\mathrm{BMX}$ down-regulated cell sensitivity to ABC294640 in ABC294640resistant cells. Further, knockdown of BMX enhanced the effect of ABC294640 treatment on tumor growth both in vitro and in vivo. These findings suggest that BMX upregulation contributes to the resistance of CRC to ABC294640 therapy.

\section{CONCLUSION}

These findings demonstrate that BMX upregulation impedes the antitumor effect of ABC294640 in CRC cells, and knockdown of BM augments $\mathrm{ABC} 294640$ ability to suppress the tumor growth of CRC in vitro and in vivo. Therefore, these data may present a novel therapeutic strategy for the CRC therapy using a combination of ABC294640 treatment and BMX blockade.

\section{DECLARATIONS}

\section{Conflict of Interest}

The authors declare that no conflict of interest is associated with this work.

\section{Contribution of authors}

We declare that this work was done by the authors named in this article and all liabilities pertaining to claims relating to the content of this article will be borne by the authors. Zhoufeng Chen designed all the experiments and revised the paper. Ruifang Jin and Haibo Xue performed the experiments, Ruifang Jin and Mengjun Chen wrote the paper.

\section{REFERENCES}

1. Aziz MA, Yousef $Z$, Saleh AM, Mohammad S, Al Knawy $B$. Towards personalized medicine of colorectal cancer. Crit Rev Oncol Hematol 2017; 118: 70-78.

2. Kavousipour $S$, Khademi $F$, Zamani M, Vakili $B$, Mokarram $P$. Novel biotechnology approaches in colorectal cancer diagnosis and therapy. Biotechnol Lett 2017; 39(6): 785-803.

3. Bever KM, Le DT. An Expanding Role for Immunotherapy in Colorectal Cancer. J Natl Compr Canc Netw 2017; 15(3): 401-410.

4. Ogretmen B. Sphingolipid metabolism in cancer signalling and therapy. Nat Rev Cancer 2017.

5. Zhang L, Urtz N, Gaertner F, Legate KR, Petzold T, Lorenz M, Mazharian A, Watson SP, Massberg S. Sphingosine kinase 2 (Sphk2) regulates platelet biogenesis by providing intracellular sphingosine 1 phosphate (S1P). Blood 2013; 122(5): 791-802.

6. Shi $W N$, Cui $S X$, Song $Z Y$, Wang $S Q$, Sun $S Y, Y u X F, L i$ $Y$, Zhang $Y H$, Gao ZH, Qu XJ. Overexpression of SphK2 contributes to ATRA resistance in colon cancer through rapid degradation of cytoplasmic RXRalpha by K48/K63-linked polyubiquitination. Oncotarget 2017; 8(24): 39605-39617.

7. Liu H, Toman RE, Goparaju SK, Maceyka M, Nava VE, Sankala H, Payne SG, Bektas M, Ishii I, Chun J, et al. Sphingosine kinase type 2 is a putative $B H 3$-only protein that induces apoptosis. J Biol Chem 2003; 278(41): 40330-40336.

8. Sun E, Zhang W, Wang L, Wang A, Ma C, Lei M, Zhou X, Sun $Y$, Lu B, Liu L, et al. Down-regulation of Sphk2

Trop J Pharm Res, May 2018; 17(5): 765 
suppresses bladder cancer progression. Tumour Biol 2016; 37(1): 473-478.

9. Gao $P$, Smith $C D$. Ablation of sphingosine kinase-2 inhibits tumor cell proliferation and migration. Mol Cancer Res 2011; 9(11): 1509-1519.

10. Dai L, Bai A, Smith $C D$, Rodriguez $P C, Y u F$, Qin Z. $A B C 294640$, a novel sphingosine kinase 2 inhibitor induces oncogenic virus infected cell autophagic death and represses tumor growth. Mol Cancer Ther 2017.

11. Lewis CS, Voelkel-Johnson C, Smith CD. Suppression of $c-M y c$ and RRM2 expression in pancreatic cancer cells by the sphingosine kinase-2 inhibitor $A B C 294640$. Oncotarget 2016; 7(37): 60181-60192.

12. Chau CH, Clavijo CA, Deng HT, Zhang Q, Kim KJ, Qiu Y, Le AD, Ann DK. Etk/Bmx mediates expression of stressinduced adaptive genes VEGF, PAI-1, and iNOS via multiple signaling cascades in different cell systems. Am J Physiol Cell Physiol 2005; 289(2): C444-C454.

13. Zhang J, Ping $P$, Wang GW, Lu M, Pantaleon D, Tang $X L$, Bolli R, Vondriska TM. Bmx, a member of the Tec family of non-receptor tyrosine kinases, is a novel participant in pharmacological cardioprotection. Am J Physiol Heart Circ Physiol 2004; 287(5): H2364-2366.

14. Cenni B, Gutmann S, Gottar-Guillier M. BMX and its role in inflammation, cardiovascular disease, and cancer. Int Rev Immunol 2012; 31(2): 166-173.

15. Holopainen $T$, Lopez-Alpuche $V$, Zheng $W$, Heljasvaara $R$, Jones $D$, He $Y$, Tvorogov D, D'Amico G, Wiener $Z$, Andersson LC, et al. Deletion of the endothelial Bmx tyrosine kinase decreases tumor angiogenesis and growth. Cancer Res 2012; 72(14): 3512-3521.

16. Wei T, Zhu W, Fang S, Zeng $X$, Huang J, Yang J, Zhang $J$, Guo L. miR-495 promotes the chemoresistance of SCLC through the epithelial-mesenchymal transition via Etk/BMX. Am J Cancer Res 2017; 7(3): 628-646.

17. Peng J, Wang Q, Liu H, Ye M, Wu X, Guo L. EPHA3 regulates the multidrug resistance of small cell lung cancer via the PI3K/BMX/STAT3 signaling pathway. Tumour Biol 2016; 37(9): 11959-11971.

18. Livak KJ, Schmittgen TD. Analysis of relative gene expression data using real-time quantitative PCR and the 2(-Delta Delta C(T)) Method. Methods 2001; 25(4): 402-408.

19. Surriga O, Rajasekhar VK, Ambrosini G, Dogan Y, Huang $R$, Schwartz GK. Crizotinib, a c-Met inhibitor, prevents metastasis in a metastatic uveal melanoma model. Mol Cancer Ther. 2013; 12(12): 2817-26.

20. Dany M. Sphingosine metabolism as a therapeutic target in cutaneous melanoma. Trans/ Res 2017; 185: 1-12.

21. Britten $C D$, Garrett-Mayer E, Chin $S H$, Shirai $K$, Ogretmen B, Bentz TA, Brisendine A, Anderton $K$, Cusack SL, Maines LW, et al. A Phase I Study of ABC294640, a First-in-Class Sphingosine Kinase-2 Inhibitor, in Patients with Advanced Solid Tumors. Clin Cancer Res 2017; 23(16): 4642-4650.

22. Xun C, Chen MB, Qi L, Tie-Ning Z, Peng X, Ning L, ZhiXiao C, Li-Wei W. Targeting sphingosine kinase 2 (SphK2) by ABC294640 inhibits colorectal cancer cell growth in vitro and in vivo. J Exp Clin Cancer Res 2015; 34: 94.

23. Guo S, Sun F, Guo Z, Li W, Alfano A, Chen H, Magyar CE, Huang J, Chai TC, Qiu S, et al. Tyrosine kinase $E T K / B M X$ is up-regulated in bladder cancer and predicts poor prognosis in patients with cystectomy. PLoS One 2011; 6(3): e17778.

24. Dai B, Kim O, Xie Y, Guo Z, Xu K, Wang B, Kong X, Melamed J, Chen $H$, Bieberich $C J$, et al. Tyrosine kinase Etk/BMX is up-regulated in human prostate cancer and its overexpression induces prostate intraepithelial neoplasia in mouse. Cancer Res 2006; 66(16): 8058-8064.

25. Fox JL, Storey A. BMX Negatively Regulates BAK Function, Thereby Increasing Apoptotic Resistance to Chemotherapeutic Drugs. Cancer Res 2015; 75(7): 1345-1355.

26. Xue LY, Qiu Y, He J, Kung HJ, Oleinick NL. Etk/Bmx, a $\mathrm{PH}$-domain containing tyrosine kinase, protects prostate cancer cells from apoptosis induced by photodynamic therapy or thapsigargin. Oncogene 1999; 18(22): 33913398. 